\title{
Robust Digital Image Watermarking Scheme in Discrete Wavelet Transform domain using Support Vector Machines
}

\author{
B.Jagadeesh \\ Associate Professor \\ E.C.E. Department \\ G.V.P. College of Engg. \\ Visakhapatnam, A.P., India.
}

\author{
P.Rajesh Kumar \\ Associate Professor \\ E.C.E. Department \\ Andhra University \\ Visakhapatnam, A.P., India.
}

\author{
P.Chenna Reddy \\ Professor \\ C.S.E. Department \\ JNTUA College of Engg. \\ Pulivendula, Kadapa, India
}

\begin{abstract}
This paper presents a robust and blind watermarking scheme for copyright protection of images in discrete wavelet transform domain based on the support vector machines (SVMs). This scheme is based on the relation between the coefficients in various sub bands in discrete wavelet transform decomposition. The proposed scheme is very secured and robust to various attacks, viz., Low pass Filtering, Salt \& Pepper noise, Gamma Correction, JPEG Compression, Row-Column Copying, Row-column blanking, Bit plane removal, Cropping, Resize and Histogram Equalization etc. Experimental results show that the proposed scheme has significant improvements in both robustness and imperceptibility and superior to an algorithm proposed by $\mathrm{Li}$ et al. in terms of Normalized Cross correlation (NC) and Peak Signal to Noise Ratio (PSNR).
\end{abstract}

\section{KEYWORDS}

Digital Image Watermarking, Discrete Wavelet Transform, Support Vector Machines.

\section{INTRODUCTION}

Digital Image watermarking is one of the proposed solutions for copyright protection of digital images. The process of embedding a watermark i.e. (Image or pseudo random sequence) in a multimedia object is termed as watermarking [1]. This watermark is embedded through invisible means in host image so that it can be extracted as the evidence of rightful ownership, when required. Once the watermark is embedded several image processing attacks may be experienced because the multimedia object can be digitally processed. In order to enhance the precision, robustness and security of watermark, many scholars have researched artificial intelligence and machine learning methods. Support Vector Machines (SVMs) are a set of supervised learning methods proposed by Vapnik et al. in the mid of 1990s, which is based on statistical learning theory and the Vapnik-Chervonenkis (VC) dimension [2]. Image watermarking algorithms which are based on the machine learning theory [3-7] are available in the literature.

$\mathrm{Fu}$ et al. [8] proposed an SVM-based watermarking method in which the difference of the intensity level of pixels of blue components was used to train the SVM. Tsaia et al. [9] presented a robust lossless watermarking algorithm based on $\alpha$-trimmed mean algorithm and support vector machines (SVMs), in which the SVM is trained to memorize the relationship between the watermark and image-dependent watermark other than inserting watermark into the host image. Li et al. [10] introduced a semi-fragile watermarking algorithm based on SVM's. This algorithm first gives the definition of wavelet coefficient direction tree, then a relation mathematical model between root node and its offspring nodes is established using SVM and further watermark is embedded and extracted based on this structuring data using relation (relational model). Hong et al. [11] proposed a novel image watermarking method in multiwavelet domain based on support vector machines (SVMs), in which the special frequency band and property of image in multiwavelet domain are employed for the watermarking algorithm.

In this paper, a modified watermarking method using discrete wavelet transform and support vector machines for embedding and extracting the watermark based on an algorithm proposed by $\mathrm{Li}$ et al. [10] is presented. This scheme is based on the relation between the root coefficients and offspring coefficients in discrete wavelet transform decomposition and the corresponding sub bands.

This paper is organized as follows: In section 2 Preliminaries about Discrete Wavelet Transform and Support Vector Machine are described. Section 3 explains the proposed watermarking method. Experimental results are shown in section 4. The conclusions are specified in section 5 .

\section{Preliminaries}

\subsection{Discrete Wavelet Transform}

The transformation of an image from the spatial domain to the frequency domain by passing it through a series of low-pass filters and high-pass filters is done by a two-dimensional DWT. The outputs of such filters correspond to multi-resolution sub-bands each possessing unique characteristics making it suitable for specific digital image processing applications. The decomposition of each level produces four bands of data denoted by LL, HL, LH, and HH. To obtain another level of decomposition LL subband can further be decomposed. This process is continued until the preferred number of levels determined by the application is reached.

The wavelet coefficients across different decomposition levels are correlated based on the spatialfrequency characteristics of wavelet transformation. This 
can be described as parent-children relationship as shown in Figure 1. For example, here one coefficient from $\mathrm{HL}_{3}$, 4 coefficients from $\mathrm{HL}_{2}$, and 16 coefficients from $\mathrm{HL}_{1}$ correspond to the same spatial location, so they are closely correlated.

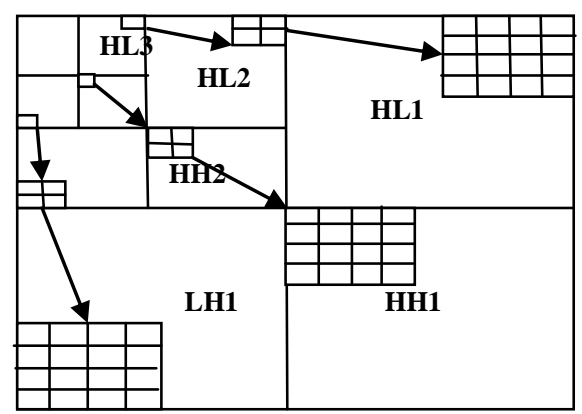

Figure 1. Demonstration of root node and offspring nodes

A direction tree is a set of coefficients from the same orientation but different decomposition levels corresponding to the same spatial location, denoted as Dij. Therefore, for an image of size $\mathrm{M} / 2^{\mathrm{m}} \times \mathrm{N} / 2^{\mathrm{m}}$ there are a total of $\mathrm{M} \mathrm{X} \mathrm{N} \mathrm{direction} \mathrm{trees,} \mathrm{each} \mathrm{direction} \mathrm{tree}$ can be used to hide at least one bit watermark. So, in the same direction tree, there exists a very close relation among coefficients and any modifications made on the image will affect this relation.

\subsection{Support Vector Machines (SVMs)}

The support vector machines (SVMs) is a machine learning tool or a universal classification algorithm used for performing classification and detection tasks. SVM has been successfully applied to pattern recognition problems and numerous classifications such as image recognition, text categorization and bioinformatics. The classifier based on SVM is used to minimize the structural misclassification risk, whereas the classification based on conventional techniques often produce minimization of the empirical risk, for that reason, SVM is claimed to lead improved generalization properties. Further, for a classification problem appliance of SVM outcome in the worldwide solution. As the efficiency do not directly depends on the dimension of the classified entities, SVM-based classification is more attractive. The number of error classification features do not have to be radically limited, so this property is very useful in fault diagnostics.

In SVM algorithm two sets of vectors are considered, one is of real numbers and the other is output vector consisting of positive and negative examples. In order to minimize the number of errors, a machine to learn the mapping from input to output is to be constructed. Hyper plane is the separating plane between positive and negative examples.

A classification task usually involves with training and testing data consisting of some data instances, where each instance in the training set contains one "target value (class labels)" and several "attributes (features)". The final goal of SVM is to produce a model by predicting a target value of data instances in the testing set which are given only the attributes.
In general SVM's are used to learn the mapping between training set and positive and negative values. To learn the mapping: $X € Y$ where $\mathrm{x} \in \mathrm{X}$ is some object and $y € Y$ is a class label. Let's take the simplest case 2-class classification, so: $\mathrm{x} € \mathrm{R}, \mathrm{y} €\{+$ or(-)1\}.A classifier function is defined as $\mathrm{y}=\mathrm{f}(\mathrm{x}, \alpha)$, where $\alpha$ are the parameters of the function in other words it can be written as $f(x,\{w, b\})=\operatorname{sign}(w \cdot x+b)$. where $w$ and $b$ are some constants used to determine the test errors.

Training and Test errors: Training error is also called as empirical risk .which is given by the equation.

$$
\mathrm{R}_{\mathrm{emp}}(\alpha)=\frac{1}{\mathrm{~m}} \sum_{\mathrm{i}=1}^{\mathrm{m}} l(\mathrm{f}(\mathrm{xi}, \alpha), \mathrm{yi})=\text { Training Error }
$$

Test error $\leq$ Training error + complexity of set of models. Complexity gives an idea about the number of training sets involved in the mapping. To reduce the test error the complexity or the capacity function should be minimized.

The general form of the classifier function is $f(x i, \alpha)=$ $\mathrm{W} . \mathrm{xi}+\mathrm{b}$ and is shown in Figure 2.

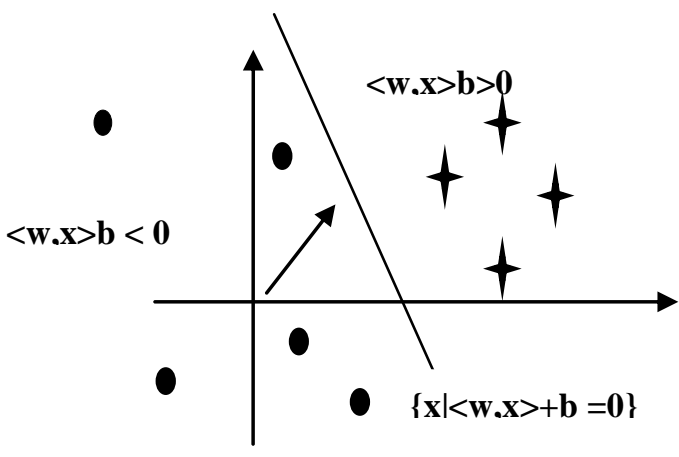

Figure 2. SVM Classifier

Requirements of SVM:

Support Vector Machine is used to the extracted image in order to enhance the quality of the image. It supports the requirements of the watermarking image like

Imperceptibility: If we apply SVM to the detected watermarks, the obtained image, after applying SVM is same as watermark image.

Efficiency: SVM supports the Efficiency by selecting the appropriate pixels during the predictions. The efficiency of the SVM increases with the increase of the database providing for the machine learning.

\section{The Proposed MeThod}

\subsection{WATERMARK EMBEDDING}

The procedure for embedding the watermark is as follows:

1. The host image of size $512 \times 512$ pixels and the watermark image of size $64 \times 64$ pixels.

2. Scramble the original watermark image $\mathrm{W}_{\mathrm{ij}}$ according to the secret key $\mathrm{K}_{1}$.

3. Perform 2-level 2D DWT on host image.

4. Randomly generate $64 \times 64$ coefficients from the sub bands in host image called root coefficients $R_{i j}$.

5. Construct the corresponding direction tree coefficients called offspring coefficients Dij. 
6. Generate the output coefficients $X_{\mathrm{ij}}$ using SVM (training, testing and prediction) with inputs as offspring coefficients $\mathrm{D}_{\mathrm{ij}}$.

7. The embedding operation is done as

$$
\mathrm{R}_{\mathrm{ij}}=\max \left(\mathrm{R}_{\mathrm{ij}}, \mathrm{X}_{\mathrm{ij}}+\beta\right) \text {, if } \mathrm{W}_{\mathrm{ij}}=1
$$$$
=\min \left(R_{i j}, X_{i j}-\beta\right) \text {, otherwise }
$$

8. Inverse DWT is applied to reconstruct the watermarked image.

\subsection{WATERMARK EXTRACTION}

The watermark extraction process from a watermarked image is as follows:

1. Perform 2-level 2D-DWT on watermarked image.

2. Randomly generate $64 \times 64$ coefficients from the sub bands in watermarked image called root coefficients Rij.

3. Construct the corresponding direction tree coefficients called offspring coefficients $D_{i j}$ '.

4. Generate the output coefficients $X_{\mathrm{ij}}$ ' using SVM

(training, testing and prediction) with inputs as offspring coefficients $\mathrm{D}_{\mathrm{ij}}$ '.

5. After calculating the output of SVM X $\mathrm{X}_{\mathrm{ij}}$ ', scrambled watermark $\mathrm{W}_{\mathrm{ij}}$ ' can be obtained as

$$
\begin{aligned}
\mathrm{W}_{\mathrm{ij}}{ }^{\prime} & =1, \text { if } \mathrm{R}_{\mathrm{ij}}{ }^{\prime}>\mathrm{X}_{\mathrm{ij}}{ }^{\prime} \\
& =0, \text { otherwise }
\end{aligned}
$$

6. Original watermark can be obtained after descrambling according to the secret key $\mathrm{K}_{1}$.

The performance metrics used to test the proposed algorithm are Peak Signal to Noise Ratio (PSNR) and Normalized Cross correlation (NC). Let the host image of size $N x N$ is $f(i, j)$ and the watermarked counterpart is $F(i, j)$, then PSNR in $\mathrm{dB}$ is given by

$$
\mathrm{PSNR}=10 \log _{10}\left(\frac{\sum_{i=1}^{N} \sum_{j=1}^{N}(F(i, j))^{2}}{\sum_{i=1}^{N} \sum_{j=1}^{N}(f(i, j)-F(i, j))^{2}}\right)
$$

Let the watermark image is denoted by $w(i, j)$ and the extracted watermark is denoted by $w^{\prime}(i, j)$ then $\mathrm{NC}$ is defined as

$$
\left.\begin{array}{l}
\mathrm{NC}= \\
\left(\sum_{i=1}^{N} \sum_{j=1}^{N}\left(w(i, j)-w_{\text {mean }}\right)\left(w^{\prime}(i, j)-w^{\prime} \text { mean }\right)\right. \\
\sqrt{\sum_{i=1}^{N} \sum_{j=1}^{N}\left(w(i, j)-w_{\text {mean }}\right)^{2} \sum_{i=1}^{N} \sum_{j=1}^{N}\left(w^{\prime}(i, j)-w^{\prime} \text { mean }\right)^{2}}
\end{array}\right)
$$

In Eq.(2), $w_{\text {mean }}$ and $w^{\prime}$ mean indicate the mean of the original watermark image and extracted watermark image respectively.

\section{EXPERIMENTAL RESULTS AND DISCUSSION}

Experiments are performed to evaluate the effectiveness of the method using host grey-scale images 'LENA', 'GOLDHILL' and 'PEPPERS' shown in Figure 3.

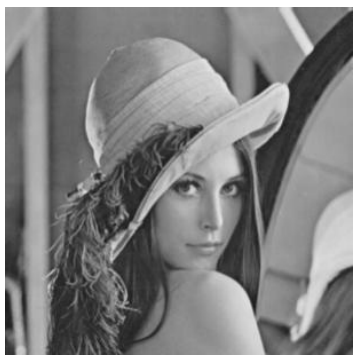

(a)

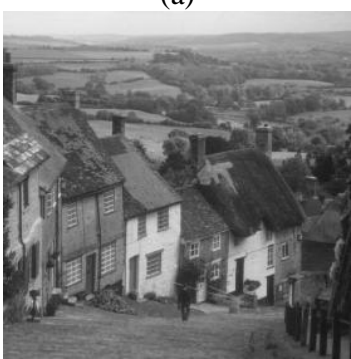

(b)

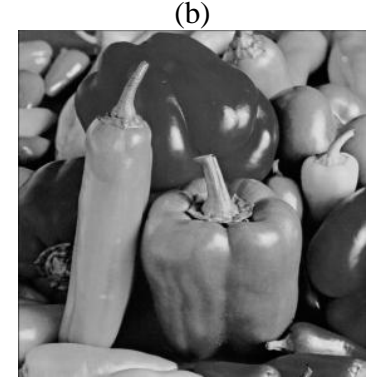

(c)

Figure.3. 512x512 (a) Lena, (b) Goldhill and (c) Peppers (Host Images)

The sizes of the host images are $512 \times 512$. The watermark image is $64 \times 64$, a logo having the letters 'JNTUACEA' as shown in Figure 4.

\section{JNTU ACEA}

\section{Figure.4. Watermark Image}

In Figure 5 watermarked LENA, GOLDHILL and PEPPERS are shown. 


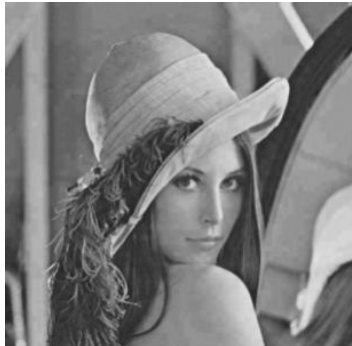

(a)

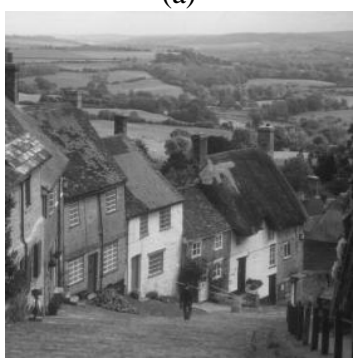

(b)

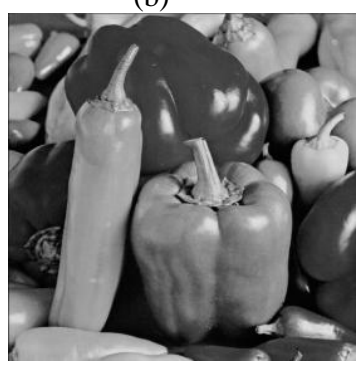

(c)

Figure. 5. 512x512 Watermarked (a) Lena (45.15dB), (b) Goldhill (42.39dB) and (c) Peppers (44.59dB)

The various attacks that are used to test the robustness of the watermark are Low pass Filtering, Salt \& Pepper noise, Gamma Correction, JPEG Compression, Row-Column Copying, Row-column blanking, Bit plane removal, Cropping, Resize and Histogram Equalization. All the attacks were tested using MATLAB 7.14.0.
For a Low pass Filtering attack a $3 \times 3$ mask that consists of 0.9 intensity values are used. The recovered watermark image and $\mathrm{NC}$ values are shown in Table.4 that shows its resilience to low pass filtering attack. The watermarked image is also attacked by salt $\&$ pepper noise with a noise density of 0.001 . The watermarked image is compressed with the use lossy JPEG compression. The index specified in the JPEG compression ranges from 0 to 100 , where 0 is finest compression and 100 is finest quality. In the row-column blanking attack, a few set of rows and columns are deleted. In row-column copy attack, a set of rows and columns are copied to the adjacent or random locations. In this attack $10^{\text {th }}$ row values is copied to $30^{\text {th }}$ row, 40 into 70,100 into 120 and $140^{\text {th }}$ row is copied into $160^{\text {th }}$ row.

In resizing attack, at first the watermarked image is reduced from $512 \times 512$ size to $400 \times 400$. By using the bicubic interpolation the dimensions are increased to $512 \times 512$. Finally, the proposed algorithm also is resistant to cropping, biplane removal, and gamma correction and histogram equalization attacks, as shown in Table 4.

The Peak Signal to Noise Ratio (PSNR) and the Normalized Cross correlation (NC) are used as a metric to compare the imperceptibility and robustness respectively are summarized in Table 1, 2 and 3 . Extracted watermarks from the watermarked image under various attacks are shown in Table 4.

Table. 1 The PSNR and NC values for Lena with Li et al.'s method and the proposed method

\begin{tabular}{|l|l|l|l|l|}
\hline \multirow{2}{*}{ Type of Attack } & \multicolumn{2}{|c|}{ Li et al.'s method } & \multicolumn{2}{c|}{ Proposed method } \\
\cline { 2 - 5 } & PSNR (dB) & NC Value & PSNR (dB) & NC Value \\
\hline No attack & 45.09 & 1 & 45.15 & 1 \\
\hline $\begin{array}{l}\text { Low pass Filtering } \\
\text { (3x3 Kernel) }\end{array}$ & 11.37 & 0.5923 & 11.38 & 0.9811 \\
\hline Salt \& Pepper Noise(0.001) & 34.60 & 0.4827 & 34.85 & 0.9634 \\
\hline Gamma Correction(0.9) & 29.01 & 0.4896 & 29.02 & 0.9525 \\
\hline $\begin{array}{l}\text { JPEG Compression } \\
\text { (QF:100) }\end{array}$ & 44.47 & 0.4920 & 44.91 & 0.9300 \\
\hline Row-Column copying & 32.01 & 0.8931 & 31.98 & 0.9028 \\
\hline Row-Column blanking & 21.35 & 0.4293 & 21.37 & 0.8025 \\
\hline Bit plane removal & 44.33 & 0.4803 & 44.38 & 0.8557 \\
\hline Cropping & 8.07 & 0.3222 & 8.08 & 0.6551 \\
\hline Resize(512-400-512) & 38.00 & 0.3339 & 38.01 & 0.4561 \\
\hline Histogram Equalization & 18.62 & 0.3499 & 18.63 & 0.5611 \\
\hline
\end{tabular}


Table. 2 The PSNR and NC values for Goldhill with Li et al.'s method and the proposed method

\begin{tabular}{|l|l|l|l|l|}
\hline \multirow{2}{*}{ Type of Attack } & \multicolumn{2}{|c|}{ Li et al.'s method } & \multicolumn{2}{c|}{ Proposed method } \\
\cline { 2 - 5 } & PSNR (dB) & NC Value & PSNR (dB) & NC Value \\
\hline No attack & 42.29 & 1 & 42.39 & 1 \\
\hline $\begin{array}{l}\text { Low pass Filtering } \\
\text { (3x3 Kernel) }\end{array}$ & 11.66 & 0.8183 & 11.67 & 0.9397 \\
\hline Salt \& Pepper Noise(0.001) & 34.10 & 0.4731 & 34.13 & 0.9699 \\
\hline Gamma Correction(0.9) & 28.23 & 0.4912 & 28.32 & 0.9746 \\
\hline $\begin{array}{l}\text { JPEG Compression } \\
\text { (QF:100) }\end{array}$ & 42.23 & 0.4918 & 42.25 & 0.9679 \\
\hline Row-Column copying & 34.23 & 0.9004 & 34.25 & 0.9330 \\
\hline Row-Column blanking & 19.76 & 0.4543 & 19.77 & 0.8777 \\
\hline Bit plane removal & 41.72 & 0.4619 & 41.75 & 0.8827 \\
\hline \multicolumn{1}{|c|}{ Cropping } & 8.33 & 0.2146 & 8.36 & 0.3077 \\
\hline Resize(512-400-512) & 34.30 & 0.3053 & 34.33 & 0.4121 \\
\hline Histogram Equalization & 16.81 & 0.3821 & 16.82 & 0.6013 \\
\hline
\end{tabular}

Table. 3 The PSNR and NC values for Peppers with Li et al.'s method and the proposed method

\begin{tabular}{|l|l|l|l|l|}
\hline \multirow{2}{*}{ Type of Attack } & \multicolumn{2}{|c|}{ Li et al.'s method } & \multicolumn{2}{c|}{ Proposed method } \\
\cline { 2 - 5 } & PSNR (dB) & $\begin{array}{l}\text { NC } \\
\text { Value }\end{array}$ & $\begin{array}{l}\text { PSNR } \\
(\mathrm{dB})\end{array}$ & NC Value \\
\hline No attack & 44.49 & 1 & 44.59 & 1 \\
\hline $\begin{array}{l}\text { Low pass Filtering } \\
\text { (3x3 Kernel) }\end{array}$ & 11.70 & 0.9490 & 11.70 & 0.9711 \\
\hline Salt \& Pepper Noise(0.001) & 34.02 & 0.4861 & 34.16 & 0.9634 \\
\hline Gamma Correction(0.9) & 28.81 & 0.4822 & 28.82 & 0.9265 \\
\hline $\begin{array}{l}\text { JPEG Compression } \\
\text { (QF:100) }\end{array}$ & 44.30 & 0.4832 & 44.35 & 0.9304 \\
\hline Row-Column copying & 30.64 & 0.9102 & 30.62 & 0.9202 \\
\hline Row-Column blanking & 21.04 & 0.4421 & 21.04 & 0.8144 \\
\hline Bit plane removal & 43.51 & 0.4488 & 43.53 & 0.8203 \\
\hline \multicolumn{1}{|c}{ Cropping } & 8.44 & 0.3223 & 8.45 & 0.6547 \\
\hline Resize(512-400-512) & 35.23 & 0.3541 & 35.26 & 0.4484 \\
\hline Histogram Equalization & 17.65 & 0.3509 & 17.67 & 0.5588 \\
\hline
\end{tabular}


Table. 4 Extracted Watermarks from the watermarked image

\begin{tabular}{|c|c|c|c|}
\hline \multirow{2}{*}{ Type of attack } & \multicolumn{3}{|c|}{ Watermarked image Type } \\
\hline & Lena & Goldhill & Peppers \\
\hline \multirow[t]{2}{*}{$\begin{array}{l}\text { Low pass Filtering } \\
\quad(3 \times 3 \text { Kernel })\end{array}$} & $\begin{array}{l}\mathrm{JN} \\
\mathrm{AC}\end{array}$ & $\begin{array}{l}\text { JNTU } \\
\text { ACEA }\end{array}$ & $\begin{array}{l}\mathrm{JN} \\
\mathrm{AC}\end{array}$ \\
\hline & NC: 0.9811 & NC: 0.9397 & NC: 0.9711 \\
\hline \multirow[t]{2}{*}{ Salt \& Pepper Noise $(0.001)$} & $\mathrm{ACEA}$ & & \\
\hline & NC: 0.9634 & NC: 0.9699 & NC: 0.9634 \\
\hline \multirow[t]{2}{*}{ Gamma Correction(0.9) } & JN'ГU. & J्ञ⿰氵工工 & $\mathbf{J} \mathbf{V}^{\prime} \mathbf{L}$ \\
\hline & NC: 0.9525 & NC: 0.9746 & NC: 0.9265 \\
\hline \multirow{3}{*}{$\begin{array}{l}\text { JPEG Compression } \\
\text { (QF:100) }\end{array}$} & J & J⿳⺈⿴囗十丁'T & J \\
\hline & $\begin{array}{l}\mathbf{A C E} \mathbf{A} \\
\therefore \quad:\end{array}$ & $\begin{array}{l}\mathbf{A C E} \\
\therefore \quad:\end{array}$ & $\begin{array}{l}\mathbf{A C E} \mathbf{A} \\
\therefore \quad:\end{array}$ \\
\hline & NC: 0.9300 & NC: 0.9679 & NC: 0.9304 \\
\hline \multirow[t]{3}{*}{ Row-Column copying } & JTTU & $\mathrm{TTT}$ & जTUं \\
\hline & $\underset{\because \because \mathbf{A}}{\mathbf{A C}}$ & $\underset{\because \mathbf{A}}{A(\mathbf{A}}$ & $\underset{\because \mathbf{A}}{\mathbf{A}}$ \\
\hline & NC: 0.9028 & NC: 0.9330 & NC: 0.9202 \\
\hline \multirow[t]{3}{*}{ Row-Column blanking } & UU & & \\
\hline & $A C A A$ & ACHA & 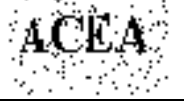 \\
\hline & NC: 0.8025 & NC: 0.8777 & NC: 0.8144 \\
\hline \multirow{3}{*}{ Bit plane removal } & 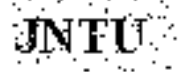 & WXIT & WTIT \\
\hline & $A C A$ & $A C H A$ & $A C H$ \\
\hline & NC: 0.8557 & NC: 0.8827 & NC: 0.8203 \\
\hline \multirow{3}{*}{ Cropping } & way & 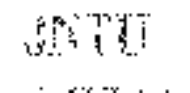 & 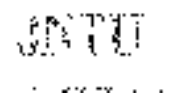 \\
\hline & 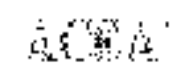 & 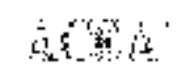 & 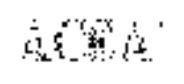 \\
\hline & NC: 0.6551 & NC: 0.6077 & NC: 0.6547 \\
\hline \multirow[t]{2}{*}{ Resize(512-400-512) } & 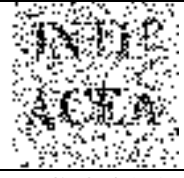 & 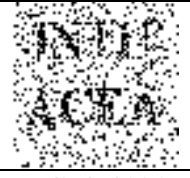 & (1) \\
\hline & NC: 0.4561 & NC: 0.4121 & NC: 0.4484 \\
\hline \multirow[t]{2}{*}{ Histogram Equalization } & 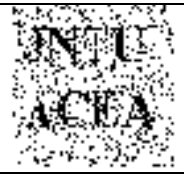 & 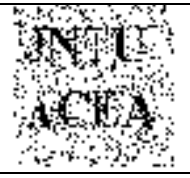 & 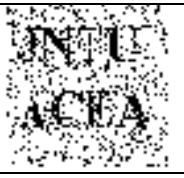 \\
\hline & NC: 0.5611 & NC: 0.6013 & NC: 0.5588 \\
\hline
\end{tabular}




\section{Conclusions}

In this paper, a robust and blind Image watermarking scheme using discrete wavelet transform based on the support vector machines have been presented. The quality of the watermarked image is fine in terms of perceptibility and PSNR. The proposed method is shown to be more robust to Low pass Filtering, Salt \& Pepper noise, Gamma Correction, JPEG Compression, Row-Column Copying, Row-column blanking, Bit plane removal, Cropping, Resize and Histogram Equalization. The test results are superior to $\mathrm{Li}$ et al.'s method in terms of $\mathrm{NC}$ values of the extracted watermarks and PSNR of the watermarked image.

\section{ACKNOWLEDGEMENTS}

Thanks to Dr.L.Venkat, G.V.P. College of Engineering, Visakhapatnam for the valuable discussions related to this work.

\section{REFERENCES}

[1] Cox, IJ, Miller, ML \& Bloom, JA, 2002, "Digital Watermarking”, Morgan Kaufmann Publisher, San Francisco, CA, USA.

[2] Steve R Gunn, 1998, "Support vector machines forclassification and regression", Technical Report, ISIS Department of electronics and computer science, University of Southampton.

[3] Wu. Jianzhen, 2009, "A RST invariant watermarking scheme utilizing support vector machine and image moments for synchronization", Fifth International Conference on Information Assurance and Security, China, pp.572-574.
[4] Xiang-Yang Wang, Zi-Han Xu, Hong-Ying Yang, 2009, "A robust image watermarking algorithm using SVM detection", Expert Sys. Appl. 36 (5), pp.9056-9064.

[5] H.H. Tsai, D.W. Sun, 2007, "Color image watermark extraction based on support vector machines", Inform. Sci. 177 (2), pp.550569.[6] P.H.H. Then, and Y.C. Wang, 2005, "Perceiving Digital Watermark Detection as Image Classification Problem using Support Vector Machine", Proc. of CITA05, pp.198-206.

[7] P.H.H. Then, and Y.C. Wang, 2006, "Support Vector Machine as Digital Watermark Detector," In Proceedings of SPIE-IS\&T Electronic Imaging, SPIE.

[8] Fu, Y., Shen, R., Lu, H., 2004, “Optimal watermark Detection based on support vector machines", Proceedings of the International Symposium on Neural Networks, Dalian, China, pp. 552-557.

[9] H.H. Tsaia, H.C. Tsenga, Y.S. Laib, 2010, "Robust lossless image watermarking based on $\alpha$-trimmed mean algorithm and support vector machine", J. Sys. Software. 83 (6), pp.1015 -1028.

[10] Chun-hua Li, Ling He-fei, Lu Zheng-ding, 2007, "Semi-fragile watermarking based on SVM for image authentication", IEEE International Conference on Multimedia and Expo, Beijing, China, pp. 1255-1258.

[11] Hong Peng, Jun Wang, Weixing Wang, 2010, "Image watermarking method in multiwavelet Domain based on support vector machine", J. Sys. Software, 83 (8) pp.1470 -1477.

[12] Colin Campbell, Yiming Ying, 2011, "Learning with Support Vector Machines", Morgan \& Claypool 